\title{
The formation of helium lines in the spectrum of COM J1740-5340
}

\author{
L. B. Lucy* \\ Astrophysics Group, Blackett Laboratory, Imperial College London, Prince Consort Road, London SW7 2AZ, UK \\ Received 7 May 2003 / Accepted 29 July 2003

\begin{abstract}
The He I $15876 \AA$ absorption line recently discovered in the spectrum of the companion to the millisecond pulsar PSR J1740-5340 is tentatively attributed to electron impact excitations due to the irradiation of its atmosphere by $\gamma$-rays emitted by the pulsar's magnetosphere. Numerical calculations, similar to those carried out previously for type Ib SNe, indicate that a pulsar beam with photon energies $\sim 1 \mathrm{MeV}$ gives rise to a $\lambda 5876 \AA$ line of the observed strength if the beam's spin-down conversion efficiency approaches $1 \%$. However, a significant difficulty for the proposed mechanism is the strength of the singlet line at $\lambda 6678 \AA$. Compared to the corresponding triplets, singlet lines are weak because of the loss of excitation when photons emitted in the series $n^{1} \mathrm{P}^{0} \rightarrow 1^{1} \mathrm{~S}$ ionize hydrogen atoms, an effect absent in the hydrogen-free atmospheres of type Ib SNe.
\end{abstract}

Key words. stars: pulsars: individual: PSR J1740-5340 - radiative transfer - stars: atmospheres - line: formation

\section{Introduction}

The binary millisecond pulsar PSR J1740-5340 was recently discovered in the globular cluster NGC 6397 by D'Amico et al. (2001a) with the Parkes radio telescope. They reported a spin period of $3.65 \mathrm{~ms}$, a 1.35 day circular orbit, and eclipses at $1.4 \mathrm{GHz}$ that last for more than $40 \%$ of the orbital period. Subsequent Parkes observations (D'Amico et al. 2001b) allowed an accurate timing solution yielding the spin-down rate as well as the binary's coordinates.

With the position known, Ferraro et al. (2001) used archived images to obtain a secure optical identification with a star (WF4-1) previously listed as a BY Dra candidate (Taylor et al. 2001). The pulsar's companion (named COM J1740-5340 by Ferraro et al. 2003) has a luminosity close to that of the cluster's main-sequence turnoff stars but its colour is anomalously red. In addition, the companion is variable with a range of $\sim 0.2 \mathrm{mag}$. This variability has the same period as the binary orbit and matches that due to the tidal distortion of a star filling its Roche lobe. Ferraro et al. (2001) emphasize that, in this regard, PSR J1740-5340 differs from two other eclipsing millisecond pulsars, where strong photometric variability is clearly due to the heating of the companions' facing hemispheres by the pulsars' emission. The absence of this expected heating effect is also reported by Kaluzny et al. (2003) and by Orosz \& van Kerkwijk (2003).

Although not apparent in broad-band light curves, the effect of the pulsar's emission on its companion has almost certainly been detected spectroscopically by Ferraro et al. (2003). From observations made to determine the spectroscopic orbit, these authors made the serendipitous discovery of He I absorption lines at 5876 and $6678 \AA$. Given that the companion's effective

^ e-mail: 1.lucy@imperial.ac.uk temperature $T_{\text {eff }} \simeq 5530 \mathrm{~K}$ (Ferraro et al. 2003) is substantially less than the $T_{\text {eff }}(\gtrsim 10000 \mathrm{~K})$ at which the HeI lines are present for main sequence stars, this discovery is a major surprise and obviously significant.

Ferraro et al. (2003) explain the presence of He I absorption in terms of a narrow strip across the illuminated face of the companion within which the local $T_{\text {eff }} \sim 10000 \mathrm{~K}$ due to heating by beamed pulsar radiation. But outside this strip, irradiation is negligible and so the local $T_{\text {eff }}$ is then $\sim 5000 \mathrm{~K}$. By a suitable choice of parameters, Ferraro et al. imply that the observed strengths of the $\lambda 5876 \AA$ line can reconciled with the absence of a photometric heating effect. In a very recent paper, this group (Sabbi et al. 2003) have published more of their spectroscopy and repeated their conclusion that the pulsar's emission pattern must be highly anisotropic.

Although the model proposed by Ferraro et al. is not implausible, another effect suggests itself if we note that COM J1740-5340 has two points in common with type Ib SNe. The first is that the spectra of these SNe are also remarkable in exhibiting He I absorption lines despite cool photospheric continua (Harkness et al. 1987).

The second point in common - the presence of high-energy photons - follows from the explanation for the helium lines in the Ib's. In the standard interpretation (Lucy 1991, hereafter Paper I; Swartz et al. 1993), a huge overpopulation of the $n=2$ levels is caused by impact excitations and ionizations of helium atoms by non-thermal electrons. These energetic electrons are the cascade products created by the multiple Compton scatterings of $\gamma$-rays emitted by the radioactive nuclei ${ }^{56} \mathrm{Co}$. There is a significant energy density of such $\gamma$-rays in the reversing layers of these $\mathrm{SNe}$ because of mass loss preceding the explosion and the mixing out of ${ }^{56} \mathrm{Ni}$ following the explosion. 
In view of these similarities, an obvious conjecture is that $\gamma$-rays emitted by the magnetosphere of PSR J1740-5340, or arising when the pulsar's relativistic wind encounters the companion's wind, irradiate the atmosphere of its companion, overpopulate the $n=2$ levels of $\mathrm{He}^{0}$, and hence give rise to detectable absorption lines. The investigation of this conjecture is the subject of this paper.

\section{An irradiated model atmosphere}

In this section, the problem is defined and then the steps needed to calculate the He line spectrum are briefly described. The discussion follows closely that given in Paper I for type Ib SNe.

\subsection{The problem}

In investigating the non-thermal excitation of helium in the atmosphere of COM J1740-5340, we face several major uncertainties. First and foremost, there is no direct evidence that PSR J1740-5340 emits a powerful beam of hard radiation. Although the object is detected in X-rays (Grindlay et al. 2001, 2002), its X-ray luminosity $L_{X}$ is weaker by a factor $\sim 17$ than expected for its spin-down luminosity $\dot{E}$ from the correlation $L_{\mathrm{X}} \sim 10^{-3} \dot{E}$ found for millisecond pulsars in the field (Becker \& Trümper 1997). However, the detected X-rays are probably not emitted from the pulsar's magnetosphere but rather from the interaction region between the pulsar's relativistic wind and the mass loss from the companion (Grindlay et al. 2002).

Despite this absence of evidence for a beam of hard radiation, we assume that such a beam exists and that it sweeps over at least part of the companion's disk. Accordingly, the problem investigated in this paper is to study the formation of the He I spectrum at a typical point on the companion's disk lying within the strip swept out by the beam.

The problem as posed refers to irradiation by a pencil beam emitted by the pulsar's magnetosphere. But the calculation is also relevant to irradiation by an extended source of hard radiation, such as that due to the colliding winds of the pulsar and its companion. The single beam then represents a one-point quadrature approximation for the effect of the extended source.

\subsection{The incident beam}

Because everything about the beam is conjectural, we take it to be mono-energetic, comprising photons of single energy $\epsilon_{0}$, the beam's effective photon energy. If the beam's flux as a function $\epsilon$ were known, $\epsilon_{0}$ could be defined as the value of $\epsilon$ such that the mono-energetic beam deposits the same fraction of its flux in the reversing layer as would the actual beam. Nevertheless, since the vertical distribution of the deposited energy would not be exact, the concept of an effective photon energy is not rigorous.

The phase-averaged flux incident on the companion's atmosphere is conveniently written as

$\mathcal{F}_{0}=\mu_{0} \times \eta \times \frac{\dot{E}}{4 \pi a^{2}} \times \frac{4 \pi}{\Omega}$ where $\mu_{0}$ is the incident direction cosine, $a$ is the orbital separation, $\eta$ is the conversion efficiency of $\dot{E}$ into beamed radiation, and $\Omega$ is the solid angle swept out by the beam.

The adopted numerical values are as follows: $\dot{E}=1.4 \times$ $10^{35} \mathrm{erg} \mathrm{s}^{-1}$ (D'Amico et al. 2001b); $a=4.33 \times 10^{11} \mathrm{~cm}$, corresponding to component masses $\mathcal{M}_{1}=1.5 \mathcal{M}_{\odot}, \mathcal{M}_{2}=0.26 \mathcal{M}_{\odot}$ (Ferraro et al. 2003); and $\Omega=1$ steradian. This last choice is standard practice in view of our ignorance concerning beaming fractions.

Inserting these values, we have $\mathcal{F}_{0}=7.48 \times$ $10^{11} \mu_{0} \eta \mathrm{erg} \mathrm{cm}^{-2} \mathrm{~s}^{-1}$.

\subsection{Model atmosphere}

A full NLTE investigation of the response of the companion's atmosphere to irradiation by a beam of $\gamma$ rays is not justified in view of the above-mentioned uncertainties. Accordingly, the approach adopted is to embed a NLTE calculation for He atoms in an atmosphere that is otherwise unperturbed by irradiation. Moreover, a severely simplified model is adopted for this unperturbed atmosphere. Specifically, a plane-parallel, Schuster-Schwarzschild model is assumed comprising a blackbody emitting photosphere at Rosseland mean optical depth $\tau=2 / 3$ and an isothermal reversing layer with temperature $T=0.93 T_{\text {eff }}$. On the assumption of hydrostatic equilibrium and constant Rosseland mean opacity $\kappa_{\mathrm{R}}$, the variation of gas pressure in the reversing layer is given by $P=\tau \times g / \kappa_{\mathrm{R}}$, where $g$ is the surface gravity. The adopted constant opacity $\kappa_{\mathrm{R}}$ is the solution of the equation $P=2 / 3 \times g / \kappa_{\mathrm{R}}\left(P, T_{\text {eff }}\right)$.

The parameters of the model atmosphere are $T_{\text {eff }}=5530 \mathrm{~K}$ (Ferraro et al. 2003), $\log g=3.44, X=0.75$ and $Y=$ 0.25 . The value of $\log g$ corresponds to radius $R_{2}=1.6 R_{\odot}$ (Orosz \& van Kerkwijk 2003) and mass $\mathcal{M}_{2}=0.26 \mathcal{M}_{\odot}$. The adopted chemical composition neglects metals since $[\mathrm{Fe} / \mathrm{H}] \sim$ -2 (Ferraro et al.) and assumes that envelope stripping has not yet exposed He-enriched zones.

With these parameters, the equation for the opacity has the solution $\kappa_{\mathrm{R}}=-1.13 \mathrm{dex}$. This was obtained by iterative interpolation in Table 6 of Alexander \& Ferguson (1994). Although this Table is computed for $Z=0.02$, the $\mathrm{H}^{-}$ion is the dominant source of opacity for the given photospheric parameters, with metals as minor contributors to the electron density $n_{\mathrm{e}}$.

In the subsequent calculations, the reversing layer is discretized into 50 layers of width $\Delta \log \tau=0.1$, within each of which physical variables are constant. The resulting small optical depths of the outermost layers ensure that line centres are accurately calculated.

\subsection{Beam loss rates}

The first step in calculating the He spectrum is to derive the rate at which the beam deposits energy in the reversing layer in the form of energetic electrons. Each incident $\gamma$-ray creates a cascade of non-thermal electrons by multiple Compton scatterings off free and bound electrons. 
At optical depth $\tau$, the rate at which $\gamma$-rays are losing energy by Compton scattering is

$\mathcal{L}(\tau)=4 \pi \int f_{\epsilon} \sigma_{\epsilon} J_{\epsilon} \mathrm{d} \epsilon$

where $\sigma_{\epsilon}$ is the Compton scattering coefficient, $f_{\epsilon}$ is the expected fraction of $\epsilon$ transferred to the Compton electron, and $J_{\epsilon}(\tau)$ is the mean intensity at photon energy $\epsilon$. Note that here, and throughout the paper, rates refer to unit volume.

As in Paper I, a Monte Carlo code is used to simulate this process. If the incident flux $\mathcal{F}_{0}$ at $\tau=0$ is represented by $\mathcal{N}$ identical photon packets containing $\gamma$-rays of energy $\epsilon_{0}$ and entering the atmosphere in time interval $\Delta t$, then each packet's initial energy $w_{0}$ is given by $\mathcal{N} \times w_{0} / \Delta t=\mathcal{F}_{0}$. These packets are followed as they propagate within the reversing layer until they escape at $\tau=0$ or penetrate below the photosphere at $\tau=2 / 3$, where they are assumed to thermalize.

When the scattering histories of all $\mathcal{N}$ packets are complete, the loss rate in the $m$ th layer is derived with the Monte Carlo estimator (Lucy 1999, 2003)

$\mathcal{L}_{m}=\frac{w_{0}}{\Delta t} \frac{1}{V} \sum \frac{w_{\epsilon}}{w_{0}} \Delta s f_{\epsilon} \sigma_{\epsilon}$.

Here $V$ is the volume of a vertical column of unit cross section through the $m$ th layer, and $\Delta s$ is the pathlength of a packet of energy $w_{\epsilon}$ between consecutive events, where an event is either a Compton scattering or a crossing of a layer's boundary. The summation is over all pathlengths in layer $m$.

Monte Carlo convergence experiments show that this estimator gives fractional errors $\lesssim 10^{-3}$ for $\mathcal{N}=10^{6}$, the sample size adopted here. Note that this estimator returns a nonzero value for the loss rate even if no $\gamma$-ray packet undergoes a Compton scattering in layer $m$.

Because $\mathcal{L}$ is proportional to $\eta$, it is convenient to compute its value $\mathcal{L}^{1}$ for $\eta=1$. The energy deposition rate in layer $m$ is then $\eta \mathcal{L}_{m}^{1}\left(\epsilon_{0}, \mu_{0}\right)$.

\subsection{Deposition fractions}

The next step is to determine the rates of impact excitations and ionizations of He atoms. To achieve this, we must follow the slowing down of fast electrons as they interact with matter in the reversing layer and record the energy transfers into the various deposition channels.

This calculation is greatly facilitated by two approximitions adopted in Paper I. First, noting that the stopping distance for an $\mathrm{MeV}$ electron is $\sim 10^{-2}$ that for an $\mathrm{MeV}$ photon (Colgate et al. 1980), we assume that the electrons deposit all their energy in situ. The second approximation (Meyerott 1978) makes use of the asymptotic near constancy of $D_{k}(E)$, the fraction of an electron's kinetic energy $E$ that is deposited in channel $k$. Thus we need only compute the deposition fractions $D_{k}^{\dagger}$ for $E^{\dagger}=10 \mathrm{keV}$ since $D_{k}(E) \simeq D_{k}^{\dagger}$ for $E>E^{\dagger}$.

The values $D_{k}^{\dagger}$ are obtained by solving the Spencer-Fano equation - Eq. (2) in Paper I. This requires that we identify the stopping mechanisms and provide their cross sections. In
Paper I, this equation was solved for a weakly-ionized pure helium gas, so that the stopping mechanisms were impact excitations and ionizations of $\mathrm{He}^{0}$ atoms and Coulomb scattering with free (thermal) electrons. Now the equation must be solved for a weakly-ionized $\mathrm{H}-\mathrm{He}$ gas, so the stopping mechanisms to be added are impact excitations and ionizations of $\mathrm{H}$ atoms.

The treatment of He impacts and Coulomb scattering remain as described in Sect. 2.3 of Paper I. The added impact cross sections for $\mathrm{H}$ are the approximate formulae of Johnson (1972), with the $\mathrm{H}$ atom represented by 14 bound levels with principal quantum numbers $n=1-14$. In addition, the probability distribution for the energy $E_{\mathrm{s}}$ of the secondary electron when $\mathrm{H}$ is ionized is taken to be $\propto\left(E_{\mathrm{s}}^{2}+J^{2}\right)^{-1}$ with $J=8 \mathrm{eV}$ (Shull 1979).

With composition specified, the deposition fractions $D_{k}^{\dagger}$ are functions of just one variable $x_{\mathrm{e}}$, the fractional ionization of H. In conformity with the assumption (Sect. 2.3) that the atmosphere is unperturbed by irradiation, electron densities are computed from Saha's equation. Then, neglecting the variation with height, we set $x_{\mathrm{e}}=x_{\mathrm{e}}^{*}=5.2 \times 10^{-5}$, its value at $\tau=1 / 3$. With this approximation, the deposition of non-thermal energy throughout the reversing layer is governed by the single vector $D_{k}^{\dagger}\left(x_{\mathrm{e}}^{*}\right)$.

Summing the appropriate elements of this vector, we find that $11.7 \%$ of the deposited energy is converted directly into heat via Coulomb scattering, $82.0 \%$ is converted into ionization and excitation energy of $\mathrm{H}$, and the remaining $6.3 \%$ is converted into ionization and excitation energy of He.

\subsection{Statistical equilibrium of helium}

The rate at which non-thermal energy is absorbed in exciting He atoms to level $i$ in layer $m$ is $\eta \mathcal{L}_{m}^{1} \times D_{i}^{\dagger}$. Accordingly, if $W_{i}$ is the energy of this level relative to the ground state, the rate of impact excitation (ionization) of level $i$ in layer $m$ is

$\Gamma_{i}=\eta \times \mathcal{L}_{m}^{1}\left(\epsilon_{0}, \mu_{0}\right) \times \frac{D_{i}^{\dagger}}{W_{i}}$.

If $\Lambda_{j i} n_{j}$ denotes the rate at which transitions $j \rightarrow i$ occur due to conventional radiative and collisional (thermal) processes, then, with impacts included, the equations of statistical equilibrium for He levels $i=2,3, \ldots, \kappa$ are

$\sum_{j=1}^{\kappa}\left(\Lambda_{j i} n_{j}-\Lambda_{i j} n_{i}\right)=-\Gamma_{i}$

When the constraint $\sum_{1}^{\kappa} n_{i}=n_{\mathrm{He}}$ is added, we have $\kappa$ equations in the $\kappa$ unknowns $n_{i}$. This system must be solved in each layer of the atmosphere in order to compute the emergent profiles of the He lines. But since the radiative coefficients in Eq. (5) depend on the solutions of the line transfer problems, iterations are required (Sect. 2.8).

The data sources for the $\Lambda$ coefficients in Eq. (5) are as stated in Paper I, except that the collision strengths of transitions between the lowest five levels of He have been updated using the calculations of Sawey \& Berrington (1993). 


\subsection{Escape probabilities}

For most of the He lines, we can assume that line photons in the reversing layer interact only with their own transition - i.e., continuum process can be neglected. But this is not true for transitions to the ground state since the emitted photons can ionize hydrogen and the atmosphere is optically thick in the Lyman continuum.

For the adopted atomic model, this is relevant for the permitted ground state transitions $\mathrm{n}^{1} \mathrm{P}^{0} \rightarrow 1^{1} \mathrm{~S}$ at 584, 537 and $522 \AA$. Since the optical depths for these transitions are also huge, we can assume that when such a line photon is emitted it is absorbed in situ either by the line itself or by a $\mathrm{H}$ atom. Accordingly, we adopt an escape probability formalism for these three transitions and only consider thermal motions in computing Doppler broadening.

If $\phi_{v}$ denotes the normalized absorption profile, a line photon emitted with frequency $v$ sees a line absorption coefficient $\ell_{v}=a_{v} n_{j}$, where

$a_{v}=\frac{\pi e^{2}}{m_{\mathrm{e}} c} f_{j i}\left(1-\frac{g_{j} n_{i}}{g_{i} n_{j}}\right) \phi_{v}$

and a continuum absorption coefficient $k_{v}=a_{\mathrm{H}}(v) n_{\mathrm{H}}$, where we assume all $\mathrm{H}$ atoms are neutral and in the ground state and neglect the correction for stimulated emission. The probability that this particular photon will be lost to the line is therefore $k_{v} /\left(k_{v}+\ell_{v}\right)$. Thus, if we average over the emission profile, the escape probability is

$\beta_{i j}=\int \frac{k_{v}}{k_{v}+\ell_{v}} \phi_{v} \mathrm{~d} v$.

Note that the emission profile is taken to be identical to the absorption profile on the assumption of complete redistribution (Mihalas 1978, p. 29).

This treatment is applied to the permitted transitions $i \rightarrow j$ for $j=1$. As a result, the radiative contribution of these transitions to Eq. (5) simplifies to $A_{i j} \beta_{i j} n_{i}$, where $A_{i j}$ is the Einstein coefficient for spontaneous emission.

For the isothermally stratified reversing layer, these escape probabilities are independent of $\tau$. With $\phi_{v}$ given by the Voigt function assuming pure radiation damping, we obtain $\beta_{i j}=$ $1.8 \times 10^{-3}, 4.4 \times 10^{-3}$ and $9.2 \times 10^{-3}$ for the lines at 584 , 537 and $522 \AA$, respectively.

An escape probability treatment is also adopted for recombinations to the ground state. The emitted photon will travel a negligible distance before it photoionizes a $\mathrm{H}$ or a $\mathrm{He}$ atom. Since the recombination photons are strongly concentrated to the He ionization threshold at $v_{T}$, the escape probability is accurately given by (Osterbrock 1974, p. 23)

$\beta_{\kappa 1}=\frac{n_{\mathrm{H}} a_{\mathrm{H}}\left(v_{\mathrm{T}}\right)}{n_{\mathrm{H}} a_{\mathrm{H}}\left(\nu_{\mathrm{T}}\right)+n_{\mathrm{He}} a_{\mathrm{He}}\left(\nu_{\mathrm{T}}\right)}$.

Accordingly, if $\alpha_{1}$ denotes the total recombination coefficient for the ground state, it is replaced in the equations of statistical equilibrium by $\beta_{\kappa 1} \alpha_{1}$. With the He threshold cross section $7.48 \times 10^{-18} \mathrm{~cm}^{2}$ of Fernley et al. (1987), we obtain $\beta_{\kappa 1}=0.68$.

\subsection{Line formation}

Subordinate transitions are not treated with escape probabilities but by solving the transfer equation. For such transitions, the radiative contribution to Eq. (5) is $A_{i j} n_{i}-\left(B_{j i} n_{j}-B_{i j} n_{i}\right) \bar{J}_{i j}$, where

$\bar{J}_{i j}=\int J_{v} \phi_{v} \mathrm{~d} v$

is the profile-averaged mean intensity. This quantity is obtained by numerical integration after solving the line transfer equation

$\mu \frac{\partial I_{v}}{\partial z}=-\ell_{v}\left(I_{v}-S\right)$

with source function

$S=\frac{2 h v^{3}}{c^{2}} /\left(\frac{g_{i} n_{j}}{g_{j} n_{i}}-1\right)$

for a grid of frequencies spanning the transition. Since high precision is not required, Eq. (10) is solved only at $\mu= \pm 1 / 2$ giving $J_{v}=\left(I_{v}^{+}+I_{v}^{-}\right) / 2$. The boundary conditions on the inward and outward beams are $I_{v}^{-}=0$ at $\tau=0$ and $I_{v}^{+}=B_{v}\left(T_{\text {eff }}\right)$ at $\tau=2 / 3$.

As in Sect. 2.7, the line profile $\phi_{v}$ is given by the Voigt function assuming pure radiation damping. But in view of the moderate optical depths of the subordinate transitions, a microturbulent contribution of $3 \mathrm{~km} \mathrm{~s}^{-1}$ is now included in the Doppler parameter.

A simple iterative procedure is adopted to obtain mutually consistent solutions of the equations of statistical equilibrium and line transfer. Equation (5) is first solved with $\bar{J}_{i j}=$ $B_{v}\left(T_{\text {eff }}\right) / 2$, its optically-thin limit. The resulting level populations are then used to obtain improved values of $\bar{J}_{i j}$ from the transfer equation. This process is repeated until the average of the absolute fractional changes of the level populations in the atmosphere are $<10^{-6}$.

When the iterations have converged, each line's emergent flux is $F_{v}(0)=I_{v}^{+}(0)$, from which its residual intensity $r_{v}=$ $F_{\nu}(0) / B_{\nu}\left(T_{\text {eff }}\right)$ and equivalent width $W_{\lambda}=\int\left(1-r_{v}\right) \mathrm{d} \lambda$ are readily computed.

\subsection{Hydrogen lines}

With only minor modifications, the code described above can also be used to compute the $\mathrm{H}$ line spectrum of the irradiated atmosphere of COM J1740-5340. The vector $D_{k}^{\dagger}\left(x_{\mathrm{e}}^{*}\right)$ already contains elements giving the fractions of $\eta \mathcal{L}_{m}^{1}$ used to excite and ionize $\mathrm{H}$ atoms. Accordingly, with the standard assumption of in situ re-absorption of Lyman- continuum and line photons, the $\mathrm{H}$ line spectrum is similarly obtained by iteratively solving the statistical equilibrium and transfer equations of Sects. 2.6 and 2.8. One point of difference is that impact ionizations of $\mathrm{H}$ are allowed to increase $n_{\mathrm{e}}$ from its LTE value.

\section{Numerical results}

In this section, the simple treatment of line formation in an irradiated atmosphere developed in Sect. 2 is applied to compute 
the strengths of He lines in the spectrum of COM J1740-5340. With atmospheric parameters fixed (Sect. 2.3), line strengths depend on $\mu_{0}, \eta$ and $\epsilon_{0}$. Selecting a typical point on the disk, we set $\mu_{0}=2 / 3$ and thus limit the investigation to exploring the atmosphere's response to a beam with conversion efficiency $\eta$ and effective photon energy $\epsilon_{0}$.

\subsection{Deposition efficiency}

Line strengths depend on $\epsilon_{0}$ only through the function $\mathcal{L}_{m}^{1}\left(\epsilon_{0}, \mu_{0}\right)$ defined in Sect. 2.4. From the Monte Carlo calculations made in determining this function, it is informative to compute the fraction of incident flux deposited in the reversing layer, the fraction deposited below the photosphere at $\tau=2 / 3$, and the fraction emerging back into space at $\tau=0$.

Figure 1 plots these deposited fractions as a function of $\epsilon_{0}$ for $\mu_{0}=2 / 3$. The behaviour of these curves is readily understood from the physics of Compton scattering. At low energies, the cross section is approximately that of Thomson scattering and a negligible fraction of the photon's energy is transferred to the Compton electron. As a result, most of the incident flux is simply reflected back into space, with only a small fraction deposited within the reversing layer.

At high energies, on the other hand, the greatly reduced Klein- Nishina cross section allows a large fraction of the incident photons to pass right through the reversing layer, so that most of the incident flux is thermalized below the photosphere. The local $T_{\text {eff }}$ is therefore increased and, in consequence, so also are temperatures in the reversing layer. In this way, a sufficiently strong beam of $\gamma$-rays with $\epsilon_{0}(\mathrm{MeV}) \gtrsim 30$ has an indirect effect on the observed line spectrum, with the $n=2$ levels of $\mathrm{He}^{0}$ being populated by the photospheric continuum and by collisions with thermal electrons. This is the circumstance envisaged by Ferraro et al. (2003).

However, for a broad range of intermediate photon energies $0.04 \lesssim \epsilon_{0}(\mathrm{MeV}) \lessgtr 10$, we see from the bell-shaped curve in Fig. 1 that a significant fraction of the incident flux is deposited in the reversing layer $(\tau<2 / 3)$ and thus directly affects line formation through impact excitations and ionizations.

\subsection{Curves of growth}

Figure 1 shows that non-thermal excitation of the He I lines is favoured when the beam's photon energy is $\simeq 1 \mathrm{Mev}$. Accordingly, we now set $\epsilon_{0}=1 \mathrm{MeV}$, since a definitive failure at this $\epsilon_{0}$ would be fatal for this interpretation.

With $\epsilon_{0}$ fixed, the single remaining parameter is $\eta$, the conversion efficiency. We now take $\eta$ to be a free parameter, postponing till later a discussion of observational and theoretical constraints on its magnitude (Sect. 4.2).

As $\eta$ increases from zero, the impact rates $\Gamma_{i}$ in Eq. (5) increase proportionately - see Eq. (4), resulting eventually in column densities for the $n=2$ levels sufficient for detectable absorption of the photospheric continuum. The simplest way of illustrating this effect is by constructing curves of growth with $\eta$ as the abscissa and dimensionless equivalent width $W_{\lambda} / \lambda$ as ordinate. Accordingly, for $\log \eta$ ranging from -5 to -0.5 , the

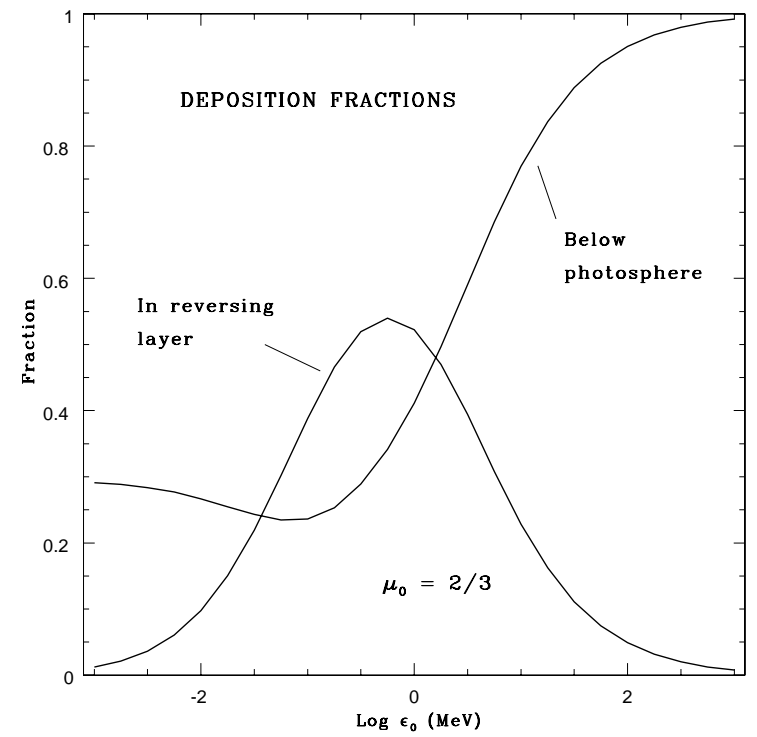

Fig. 1. Deposition of beam flux. The fractions of incident flux deposited above and below the photosphere $(\tau=2 / 3)$ are plotted against the photon energy $\epsilon_{0}$ of the mono-energetic beam. The incident direction cosine $\mu_{0}=2 / 3$.

coupled radiative transfer and statistical equilibrium problems of Sect. 2 have been solved and the resulting line profiles and equivalent widths $W_{\lambda}$ computed for all transitions represented in the adopted atomic model for $\mathrm{He}^{0}$.

Figure 2 is the curve of growth for the line HeI $\lambda 5876 \AA$. We see that $W_{\lambda}$ increases linearly with $\eta$ for $\eta \lesssim 4 \times 10^{-4}$ and thereafter more slowly. This is reminiscent of conventional curves of growth, where the linear part is followed by a flat part as the lines' Doppler cores become saturated while their damping wings remain optically thin. But here there is the additional effect of the partial filling-in of the absorption line by intrinsic line emission within the reversing layer. These additional line photons are created in the radiative cascades following impact ionizations and excitations.

The role of intrinsic emission in reducing $W_{\lambda}$ can be quantified approximately by also computing, for the given level populations, the line profile under the assumption of coherent scattering. For the adopted Schuster-Schwarzschild reversing layer, the residual intensity is then $r_{v}=1 /\left(1+0.75 N_{j} a_{v}\right)$, where $N_{j}$ is the column density of absorbing atoms and $a_{v}$ is given by Eq. (6). The curve of growth thus obtained is the dashed curve in Fig. 2. The vertical difference between the two curves increases with $\eta$, reflecting the growing contribution from cascades.

The actual line profiles used to derive these curves of growth are plotted in Fig. 3 for $\eta=10^{-2}$. In this case, intrinsic emission reduces $W_{\lambda}$ from 0.35 to $0.28 \AA$.

Since the strengths of other optical He I lines might also be relevant to testing this excitation mechanism, a selection of their curves of growth are plotted in Fig. 4. As with the $\lambda 5876 \AA$ line, all lines have curves of growth that eventually depart from linearity in reponse to the combined effects of core saturation and intrinsic emission. The most extreme case is that of the $\lambda 6678 \AA$ line, whose $W_{\lambda}$ increases to a maximum 


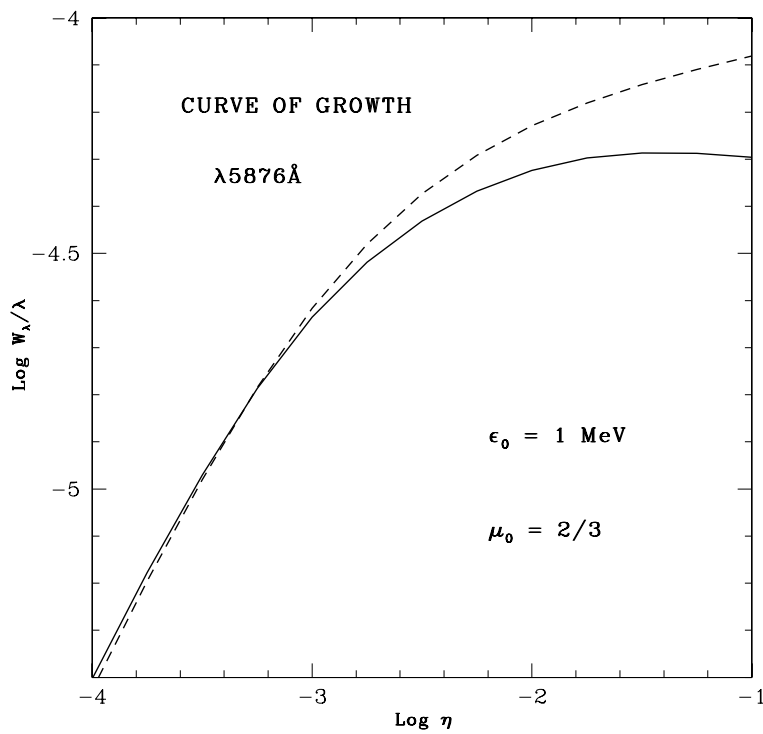

Fig. 2. Curve of growth for He I $\lambda 5876 \AA$ A. The dimensionless equivalent width $W_{\lambda} / \lambda$ is plotted against the conversion efficiency $\eta$ for the indicated beam parameters. The dashed curve corresponds to line formation by coherent scattering.

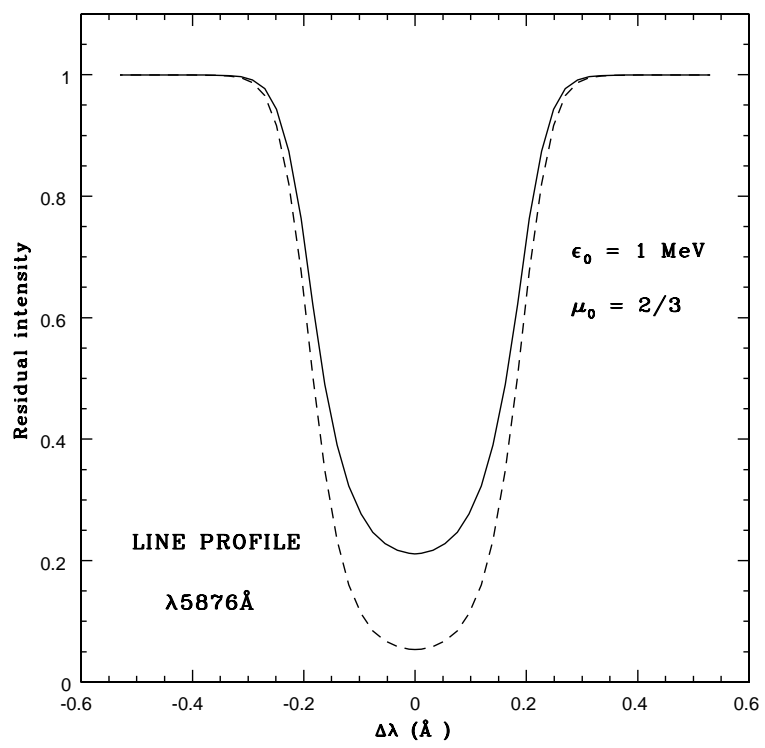

Fig. 3. Line profile of He I $\lambda 5876 \AA$ for conversion efficiency $\eta=$ 0.01 . The beam parameters are same as for Fig. 2. The dashed curve corresponds to line formation by coherent scattering.

of $\simeq 0.022 \AA$ at $\eta \simeq 5 \times 10^{-3}$ and then declines precipitously, going into emission for $\eta>1.3 \times 10^{-2}$.

\section{Comparison with observations}

In this section, the predictions of the non-thermal excitation model are compared with observational data for COM J1740-5340.

\subsection{The equivalent width of $\lambda 5876 \AA$}

The prediction that each line has a maximum $W_{\lambda}$ offers an immediate prospect of observational contradiction. From Fig. 2,

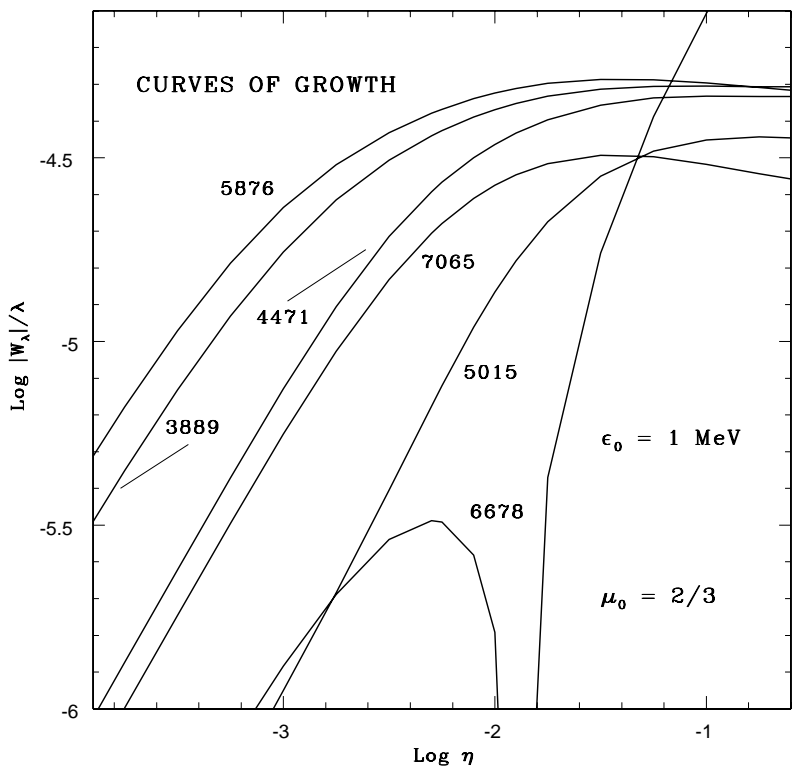

Fig. 4. Curves of growth for selected He I lines. The dimensionless equivalent widths $W_{\lambda} / \lambda$ are plotted against the conversion efficiency $\eta$. The beam parameters are same as for Fig. 2 . Note that the line $\lambda 6678 \AA$ is in emission for $\eta>0.013$.

we see that $W_{5876}$ reaches a maximum of $0.30 \AA$ at $\eta=$ $4 \times 10^{-2}$. For comparison, observed values can be estimated from the spectra published by Sabbi et al. (2003; Fig. 4). These spectra show that $W_{\lambda}$ varies with phase but reaches strengths of $\simeq 0.19 \AA$ at phases 0.02 and 0.56 .

This comparison shows that the predicted maximum exceeds observed values with a comfortable safety margin. This is just as well since, at these phases, a significant fraction of the companion's projected disk is not irradiated by the beam and is thus not expected to contribute to the integrated line profile.

Eventually, when higher $\mathrm{S} / \mathrm{N}$ spectra are obtained, it will be worthwhile to calculate line profiles integrated over the disk. This will not only give more definitive tests of line strengths but also allow the phase-dependent velocity displacement of the line centre from the orbital velocity of COM J1740-5340 to be tested. Such displacements are a consequence of only one hemisphere of the synchronously rotating companion (Ferraro et al. 2003) being irradiated. Of course, with data of exceptional quality, the technique of Doppler tomography could be used to make inferences about the pulsar's beam pattern.

\subsection{Conversion efficiency}

From Fig. 2, we find that $W_{5876}$ is comparable with the observed strengths for $\eta \gtrsim 2 \times 10^{-3}$. But this assumes the beam sweeps out solid angle $\Omega=1$ steradian (Sect. 2.2). This must be increased by a factor $\gtrsim 3.2$ for the entire facing hemisphere to be irradiated. Thus, more realistically, the required efficiency is $\eta \gtrsim 6 \times 10^{-3}$. The next question, therefore, is whether this is achievable for photon energies $\sim 1 \mathrm{MeV}$.

No data relevant to this question has yet been acquired for PSR J1740-5340. But such data is available for the binary millisecond pulsar PSR J0218+4232, whose spin 
period $(2.3 \mathrm{~ms})$ and spin-down luminosity $\left(2.5 \times 10^{35} \mathrm{erg} \mathrm{s}^{-1}\right)$ (Mineo et al. 2000) are not too different from those of PSR J1740-5340. Detections and upper limits for photon energies from $0.05-10000 \mathrm{MeV}$ are available for this pulsar. From this data, Kuiper et al. (2000) suggest that this pulsar's maximum luminosity density is reached in the COMPTEL MeV range just below the reported upper limits. Accepting this suggestion and thus interpreting the $2 \sigma$ upper limit reported for the COMPTEL $0.75-3 \mathrm{MeV}$ band as a detection, we find $\eta_{\text {obs }} \sim$ $15 \%$ for a 1 steradian beam. This is to be compared with $\eta_{\text {obs }} \sim$ $7 \%$ found by Kuiper et al. from EGRET detections for energies between $100 \mathrm{MeV}$ and $1 \mathrm{GeV}$.

This interpretation of the data for PSR J0218+4232 indicates that the efficiency required if $\epsilon_{0} \sim 1 \mathrm{MeV}$ presents no problem. But note that, for this same data set, Dyks \& Rudak (2002) offer a theoretical interpretation which predicts fluxes in the COMPTEL bands far below the reported upper limits. Specifically, at $\sim 1 \mathrm{MeV}$ their model predicts $\eta \sim 10^{-3}$, corresponding to a barely detectable line at $\lambda 5876 \AA$.

\subsection{Heating}

The 1-steradian efficiency $\eta \sim 2 \times 10^{-3}$ needed to produce the observed strength of the $\lambda 5876 \AA$ line corresponds to an incident $\gamma$-ray flux $\mathcal{F}_{0}=7.5 \times 10^{8} \mathrm{erg} \mathrm{cm}^{-2} \mathrm{~s}^{-1}$. Even if all of this were thermalized below the photosphere, the increase in $T_{\text {eff }}$ of the irradiated hemisphere is only $\sim 20 \mathrm{~K}$. Thus the proposed explanation of the $\lambda 5876 \AA$ line is consistent with the absence of a detectable photometric signature of heating (Sect. 1).

\subsection{Hydrogen lines}

Hydrogen line profiles have also been calculated as a function of $\eta$. The initial thought was that the observed strength of the $\lambda 5876 \AA$ line might be achieved simultaneously with $\mathrm{H} \alpha$ going into emission, thus explaining the star's earlier listing as a BY Dra candidate (Taylor et al. 2001). In the event, at values of $\eta$ corresponding to $\lambda 5876 \AA$ reaching its observed strength, the contribution of intrinsic emission to $\mathrm{H} \alpha$ is still neglible.

However, this is not a failure of the model since it is clear from the spectra published by Sabbi et al. (2003) that $\mathrm{H} \alpha$ emission originating close to the surface of COM J1740-5340 is not due to irradiation. This conclusion follows because the $\mathrm{H} \alpha$ emission remains stong even when most of the facing hemisphere is hidden from the observer. Most likely, as Sabbi et al. conclude, the emission has a chromospheric origin as commonly found in short-period, low mass binaries.

\subsection{The equivalent width of $\lambda 6678 \AA$}

The above discussion has focussed on He I $\lambda 5876 \AA$ since this the line from which Ferraro et al. (2003) and Sabbi et al. (2003) infer that the pulsar's beam is highly anisotropic.

Of the other He I lines, the one whose predicted strength is most likely to be in conflict with observation is the $\lambda 6678 \AA$ line, which is the analogue for the singlets of the triplet line $\lambda 5876 \AA$. Ferraro et al. (2003) report the presence of the $\lambda 6678 \AA$ line but not its strength. According to these calculations, its maximum equivalent width is only $0.022 \AA$, which is probably inconsistent with detetection.

Subsequent to the original submission of this paper, E. Sabbi (Bologna) has confirmed that the $\lambda 6678 \AA$ line is indeed reliably detected. Moreover, it reaches an equivalent width well in excess of the above maximum. Specifically, at phase 0.56 , she reports that $W_{6678} \simeq 0.19 \AA$, almost an order of magnitude larger than the predicted maximum.

The weakness of $\lambda 6678 \AA$ in these calculations contrasts with its strength in models for type Ib SNe (Paper I, Fig. 1). The origin of this difference is chemical composition. The atmospheres of type Ib SNe are hydrogen-free, so there is no loss of population from singlet levels due to the ionization of hydrogen by photons emitted by the permitted ground state transitions $\mathrm{n}^{1} \mathrm{P}^{0} \rightarrow 1^{1} \mathrm{~S}$ (Sect. 2.7).

This explanation for the predicted weakness of the $\lambda 6678 \AA$ line is confirmed by recomputing the model at $\log \eta=-2.3$ but now with the line escape probabilities of Sect. $2.7 \beta_{i \rightarrow 1}=0$. The result is a dramatic increase in $W_{6678}$ from 0.022 to $0.33 \AA$.

Interestingly, in this experiment $W_{5876}$ also increases, from 0.26 to $0.35 \AA$. The reason for this is the removal of the population decrement of the $n=2$ singlet levels relative to the corresponding triplet levels. In consequence, there is no longer a significant net collisional transfer of excitation from the triplets to the singlets.

\subsection{Other lines}

In his report, the referee F. R. Ferraro (Bologna) generously included unpublished information on other He lines. This data, as also that from E. Sabbi discussed above, is from the spectra described by Ferraro et al. (2003) and Sabbi et al. (2003).

The triplet line $\lambda 3889 \AA$ has a predicted strength close to that of the $\lambda 5876 \AA$ line - see Fig. 4 - and so should be seen were it not blended with the $\mathrm{H} 8$ Balmer line.

The triplet line $\lambda 4471 \AA$ has a predicted equivalent width of $0.06 \AA$ when $W_{5876}=0.19 \AA$, as observed at phases 0.02 and 0.56 . Though weak, a line of this strength should still be visible, but Ferraro and colleagues finds no evidence of it. This is undoubtedly a difficulty but one unlikely to be specific to the non-thermal mechanism. The reason for this is that the $\lambda 5876$ and $\lambda 4471 \AA$ lines have the same lower level and the ratio of their predicted strengths is approximately equal to the ratio of their $f$-values. Thus a similar ratio should be expected for the thermal mechanism proposed by Ferraro et al. (2003)

\section{Conclusion}

The aim of this paper has been to investigate the possibility that the formation mechanism for He I absorption lines in the spectrum of COM J1740-5340 is similar to that operating in the atmospheres of type Ib SNe. This alternative merits serious consideration in view of the powerful and remarkable constraint on the pulsar's radiation pattern that follows if the He I lines are indeed formed by conventional thermal processes.

The result of this investigation is moderately discouraging for the non-thermal interpretation but not yet a decisive 
rejection. On the positive side, the crucial line $\lambda 5876 \AA$ achieves the observed strength at seemingly feasible values of the conversion efficiency $\eta$. Moreover, the corresponding heating effect is consistent with the non-detection of any such effect photometrically.

However, despite these successes, the strengths of other HeI lines, especially $\lambda 6678 \AA$, present difficulties. The question then is whether such difficulties are fatal or can be overcome by refinement or modification of the simple model used here. Among refinements to be considered are the effect of the beam on the continuum opacity, specifically the dissociation of the $\mathrm{H}^{-}$ion by impacts (Geltman 1960), the ionization of surface layers by impacts, and the inclusion of an empirical model for the chromosphere discovered by Sabbi et al. (2003). A higher level treatment of a stellar atmosphere's response to irradiation by gamma rays is an attractive technical challenge and likely to have application to other close companions of exotic objects.

Nevertheless, given the relatively low $\mathrm{S} / \mathrm{N}$ of the spectra of COM J1740-5340 and the poorly constrained parameters of the irradiation model, it is perhaps doubtful that a decision between the thermal and non-thermal line formation mechanisms can soon be reached purely on the basis of line diagnostics. Accordingly, the prediction by Sabbi et al. (2003) that up to 1\% of the disk of COM J1740-5340 radiates with $T_{\text {eff }} \sim 10000 \mathrm{~K}$ should be tested with deep UV imaging at $\lambda \lesssim 2000 \AA$. A detection of a photometric signature of a heating effect at such wavelengths would be a decisive confirmation of the thermal mechanism and therefore of a narrowly-confined pulsar beam.

Acknowledgements. I am indebted to E. Sabbi and the referee, F. R. Ferraro, for unpublished data on He line strengths.

\section{References}

Alexander, D. R., \& Ferguson, J. W. 1994, ApJ, 437, 879

Becker, W., \& Trümper, J. 1997, A\&A, 326, 682
Colgate, S. A., Petschek, A. G., \& Kriese, J. T. 1980, ApJ, 237, L81

D’Amico, N., Lyne, A. G., Manchester, R. N., Possenti, A., \& Camilo, F. 2001a, ApJ, 548, L171

D'Amico, N., Possenti, A., Manchester, R. N., et al. 2001b, ApJ, 561, L89

Dyks, J., \& Rudak, B. 2002, to appear in Proc. of the 22nd Moriond Astrophysics Meeting, Les Arcs [astro-ph/0205222]

Fernley, J. A., Taylor, K. T., \& Seaton, M. J. 1987, J. Phys. B: At. Mol. Phys., 20, 6457

Ferraro, F. R., Possenti, A., D’Amico, N., \& Sabbi, E. 2001, ApJ, 561, L93

Ferraro, F. R., Sabbi, E., Gratton, R., et al. 2003, ApJ, 584, L13

Geltman, S. 1960, Proc. Phys. Soc., 75, 67

Grindlay, J. E., Heinke, C. O., Edmonds, P. D., Murray, S. S., \& Cool, A. M. 2001, ApJ, 563, L53

Grindlay, J. E., Camilo, F., Heinke, C. O., et al. 2002, ApJ, 581, 470

Harkness, R. P., Wheeler, J. C., Margon, B., et al. 1987, ApJ, 317, 355

Johnson, L. C. 1972, ApJ, 174, 227

Kaluzny, J., Rucinski, S. M., \& Thompson, I. B. 2003, AJ, 125, 1546

Kuiper, L., Hermsen, W., Verbunt, F., et al. 2000, A\&A, 359, 615

Lucy, L. B. 1991, ApJ, 383, 308 (Paper I)

Lucy, L. B. 1999, A\&A, 344, 282

Lucy, L. B. 2003, A\&A, 403, 261

Meyerott, R. E. 1978, ApJ, 221, 975

Mihalas D., 1978, Stellar Atmospheres, 2nd ed. (San Francisco: W. H. Freeman \& Co.)

Mineo, T., Cusumano, G., Kuiper, L., et al. 2000, A\&A, 355, 1053

Orosz, J. A., \& van Kerkwijk, M. H. 2003, A\&A, 397, 237

Osterbrock, D. E. 1974, Astrophysics of Gaseous Nebulae (San Francisco: W. H. Freeman \& Co.)

Sabbi, E., Gratton, R., Ferraro, F. R., et al. 2003, ApJ, 589, L41

Sawey, P. M. J., \& Berrington, K. A. 1993, At. Data Nucl. Data Tables, 55,81

Shull, J. M. 1979, ApJ, 234, 761

Swartz, D. A., Filippenko, A. V., Nomoto, K., \& Wheeler, J. C. 1993, ApJ, 411, 313

Taylor, J. M., Grindlay, J. E., Edmonds, P. D., \& Cool, A. M. 2001, ApJ, 553, L169 